\title{
FUSING MICROWAVE RADAR AND MICROWAVE-INDUCED THERMOACOUSTICS FOR BREAST CANCER DETECTION
}

\author{
Evgeny Kirshin \\ Borislav Oreshkin \\ Kevin Guangran Zhu \\ Milica Popović \\ Mark Coates
}

Department of Electrical and Computer Engineering, McGill University, H3A 2A7, Montreal, QC, Canada

\begin{abstract}
Microwave-based techniques for breast tumour detection rely on the inherent electrical difference between malign and healthy tissue in the microwave range. Microwave-radar and microwave-induced thermoacoustic methods both struggle when the dielectric contrast between the tumorous and background tissues is relatively small. In this work, we propose a detection technique that uses a hypothesis testing framework to fuse the information provided by these two sensor modalities and hence provide more reliable detection results in low-contrast, high-clutter environments when compared to the results that either of the techniques would provide alone.
\end{abstract}

Index Terms - Microwave imaging, UWB, breast cancer detection, hypotheses testing, sensor fusion

\section{INTRODUCTION}

Early detection of breast cancer leads to a much higher recovery rate. The X-ray mammography and Magnetic Resonance Imaging (MRI) screening techniques require expensive equipment and involve significant patient discomfort; X-ray mammography involves undesirable exposure to ionizing radiation. Microwave-based techniques have some promise as complementary modalities [1]. Microwaveradar (MR) methods measure and analyze the backscatter signal when the breast is illuminated by microwaves. Microwave-induced thermoacoustic (MIT) methods measure and process the acoustic signals induced by differential microwave heating. Both techniques rely on the difference in the dielectric properties of malign and healthy tissue and their individual performance can suffer when the dielectric contrast is low. Since the techniques rely on different physical processes (wave reflection versus heating) and measure different types of signals (microwave versus acoustic), the fusion of the information provided by the two modalities has the potential to provide significantly better detection performance.

The primary contribution of this paper is the proposal of a new microwave-based method for breast cancer detection based on jointly processing MR and MIT signals in a hypothesis-testing framework. We identify a test statistic and explain how to derive its null-hypothesis distribution for the purpose of setting a threshold. We conduct numerical simulations using structurally-realistic breast models (derived from MRI scans). These simulations explore multiple settings of the dielectric parameters and tissue properties to examine the impact of low contrast and high clutter. The simulations indicate that the proposed fusion approach can provide important improvement.

\section{PROBLEM STATEMENT}

We address the detection problem of a tumour inside a breast consisting of heterogeneous tissue (glandular, fatty, etc.). The measurement

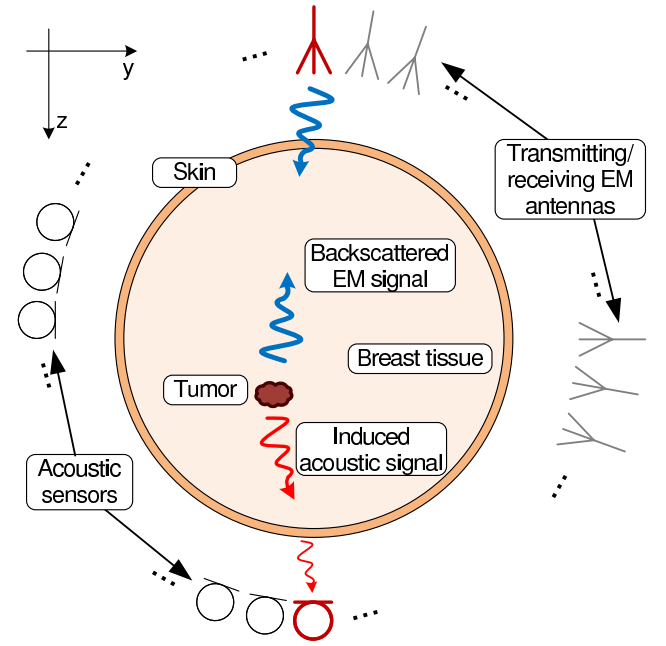

Fig. 1: A 2-D system model for the joint MR and MIT simulations.

system, depicted for a 2-D setting in Figure 1, operates in two modes. In the MR mode, a wideband microwave pulse is radiated and the respective backscattered signal is recorded sequentially at $M_{1}$ different locations (channels). In the MIT mode, modulated pulses are radiated from $M_{2}$ locations, and transducers on the opposite side of the breast measure the induced acoustic signals.

After pre-processing (see Section 4), the signals from the MR and MIT modalities form a set of column-vectors $\mathbf{x}_{i}=$ $\left[x_{i, 1}, \ldots, x_{i, N_{1}}\right]^{T}$ for $i=1 \ldots M_{1}$ and $\mathbf{y}_{j}=\left[y_{j, 1}, \ldots, y_{j, N_{2}}\right]^{T}$ for $j=1 \ldots M_{2}$ of length $N_{1}$ and $N_{2}$ respectively (we focus on the case $N_{1}=N_{2}=N$ and $M_{1}=M_{2}=M$ ). Taking into account the inherent limitations of the antenna array resolution, we model the location of the tumour as taking one of a finite set of locations $\left\{\mathbf{r}_{\ell}\right\}, \ell=1, \ldots, L$. The parameters $\boldsymbol{\theta}_{\ell}$ capture the tissue and geometry properties that affect the propagation and shape of the received signals (see Section 2.1 for a concrete example for the 2-dimensional setting). The index $\ell_{0}$ denotes the "true" location, if the tumour exists. Signals $\mathbf{x}_{i}$ and $\mathbf{y}_{i}$ can then be modeled as:

$$
\begin{aligned}
\mathbf{x}_{i} & =\beta_{\ell_{0}} \mathbf{s}_{i}\left(\boldsymbol{\theta}_{\ell_{0}}\right)+\boldsymbol{\xi}_{i}, \quad i=1, \ldots, M \\
\mathbf{y}_{j} & =\eta_{\ell_{0}} \mathbf{d}_{j}\left(\boldsymbol{\theta}_{\ell_{0}}\right)+\boldsymbol{\zeta}_{j}, \quad j=1, \ldots, M
\end{aligned}
$$

These expressions model $\mathbf{x}_{i}$ and $\mathbf{y}_{j}$ as a combination of scaled signals $\mathbf{s}_{i}\left(\boldsymbol{\theta}_{\ell_{0}}\right)$ (scattered microwave signal) and $\mathbf{d}_{j}\left(\boldsymbol{\theta}_{\ell_{0}}\right)$ (induced pressure signal) and the corresponding noise components $\boldsymbol{\xi}_{i}$ and $\boldsymbol{\zeta}_{j}$. These latter components are comprised of clutter (microwave scattering or acoustic signals generated by the normal tissue variation in the breast), as well as receiver noise and digital quantization noise.

We model the random noise vectors $\boldsymbol{\xi}_{i}$ and $\boldsymbol{\zeta}_{j}$ as jointly Gaus- 
sian with zero means and covariances $\sigma_{\xi}^{2} \mathbf{R}_{\xi}$ and $\sigma_{\zeta}^{2} \mathbf{R}_{\zeta}$, where the unit-norm matrices $\mathbf{R}_{\boldsymbol{\xi}}$ and $\mathbf{R}_{\zeta}$ capture the structural properties of the covariance, and the scalars $\sigma_{\xi}^{2}$ and $\sigma_{\zeta}^{2}$ specify the noise powers. We model the noise signals as independent among the channels and between the MR and MIT methods. We assume that $\mathbf{R}_{\boldsymbol{\xi}}$ and $\mathbf{R}_{\zeta}$ can be estimated using a training data set containing responses from a number of healthy breasts. The signal amplitudes $\beta_{\ell}$ and $\eta_{\ell}$ are treated as unknown deterministic parameters and signals $\mathbf{s}_{i}\left(\boldsymbol{\theta}_{\ell}\right)$, $\mathbf{d}_{j}\left(\boldsymbol{\theta}_{\ell}\right)$ as deterministic and dependent on a set of parameters $\boldsymbol{\theta}_{\ell}$. In addition, tissue parameters, included in $\boldsymbol{\theta}$, can be also estimated. Thus, for any given location $\mathbf{r}_{\ell}$ we consider $\mathbf{s}$ and $\mathbf{d}$ as known and refer to them as "signal templates".

We consider the binary hypothesis testing framework for the joint MR/MIT breast tumour detection formulated on the finite set of scan-locations $\left\{\mathbf{r}_{\ell}\right\}_{\ell=1}^{L}$ :

$$
\mathcal{H}_{\ell}^{(0)}: \beta_{\ell}, \eta_{\ell}=0 \text { vs. } \mathcal{H}_{\ell}^{(1)}: \beta_{\ell}, \eta_{\ell} \neq 0 .
$$

The hypothesis $\mathcal{H}_{\ell}^{(1)}$ denotes the scenario with tumour present while $\mathcal{H}_{\ell}^{(0)}$ is the null hypothesis. To discern between these hypotheses and to address the issue of unknown parameters in (1) and (2) we adopt a generalized likelihood ratio test (GLRT) approach [2].

\subsection{D Signal Templates}

For the 2-dimensional experiments described in this paper, we construct signal templates as follows. In the discussed model the set of parameters $\boldsymbol{\theta}_{\ell}$ includes average Debye tissue properties [3] (relative permittivity at the infinite frequency $\epsilon_{\infty}$, difference in the relative permittivity at DC and the infinite frequency $\Delta \epsilon$, static conductivity $\sigma_{s}$ and relaxation time constant $\tau$ ) and tumour location $\mathbf{r}_{\ell}$. The 2-D signal template for the MR process models the effect of the delay and attenuation in the matching medium, skin and tissue. Its frequency domain representation for channel $i$ is:

$$
\begin{aligned}
\mathbf{S}_{i}\left(\boldsymbol{\theta}_{\ell}\right)= & G(j \omega) e^{-j 2 k_{b}\left|\mathbf{r}_{i}-\mathbf{r}_{b, i}\right|} e^{-j 2 k_{s}\left|\mathbf{r}_{b, i}-\mathbf{r}_{s, i}\right|} \\
& \cdot e^{-j 2 k_{n}\left(\boldsymbol{\theta}_{\ell}\right)\left|\mathbf{r}_{s, i}-\mathbf{r}_{\ell}\right|},
\end{aligned}
$$

where $\mathbf{r}_{\ell}$ denotes the location of the tumour, $\mathbf{r}_{i}$ denotes the location of the $i$-th antenna; $\mathbf{r}_{b, i}$ and $\mathbf{r}_{s, i}$ lie on the line between $\mathbf{r}_{i}$ and $\mathbf{r}_{\ell}$ at the transition points of the background-skin interface and the skintissue interface respectively. $k_{b}$ and $k_{s}$ denote the known wave numbers of the matching medium and skin. $k_{n}\left(\boldsymbol{\theta}_{\ell}\right)$ is the wave number from the estimated Debye parameters. $G(j \omega)$ denotes the Gaussian modulated pulse reflected at the interface between the tissue and the tumor.

The 2-D signal template for the acoustic wave generated by the heated tumour is [4]

$$
\mathbf{D}_{i}\left(\boldsymbol{\theta}_{\ell}\right)=I(j \omega) \int_{\Omega} \frac{e^{-j k_{a}\left|\mathbf{r}_{i}-\mathbf{r}_{\ell}\right|}}{\sqrt{\left|\mathbf{r}_{i}-\mathbf{r}_{\ell}\right|}} d \Omega
$$

where $k_{a}$ denotes the acoustic wave number. $I(j \omega)$ denotes the acoustic pulse generated by a point in the tumour region $\Omega$. Note that this signal template assumes the tissue and matching medium have the same acoustic properties.

\section{METHODOLOGY}

Prior to the application of the GLRT framework, the input signals $\mathbf{x}$ and $\mathbf{y}$ are whitened using the estimated clutter covariance matrices:

$$
\tilde{\mathbf{x}}=\tilde{\mathbf{R}}_{\boldsymbol{\xi}}^{-1 / 2} \mathbf{x} ; \tilde{\mathbf{y}}=\tilde{\mathbf{R}}_{\boldsymbol{\zeta}}^{-1 / 2} \mathbf{y}
$$

The clutter covariance matrices are estimated from a training set of tumour-free breasts, using the procedure described in [5]. We set the regularization parameter to $1 \mathrm{e}-7$, a value that provides good performance in practice. In order to compensate for the distortion introduced by whitening, we expose the signal templates to the same whitening procedure, which produces $\tilde{\mathbf{s}}$ and $\tilde{\mathbf{d}}$ vectors.

The GLRT performs the comparison of the generalized likelihood ratio $L_{G}(\tilde{\mathbf{x}}, \tilde{\mathbf{y}})$ against a threshold $\gamma$ :

$$
L_{G}(\tilde{\mathbf{x}}, \tilde{\mathbf{y}}) \underset{\mathcal{H}_{\ell}^{(0)}}{\stackrel{\mathcal{H}_{\ell}^{(1)}}{\gtrless}} \gamma
$$

The generalized likelihood ratio can be factorized under the noise independency assumption:

$$
L_{G}(\tilde{\mathbf{x}}, \tilde{\mathbf{y}})=\frac{p\left(\tilde{\mathbf{x}} \mid \hat{\beta}_{\ell_{1}}, \hat{\sigma}_{\boldsymbol{\xi}, \ell_{1}}^{2}, \mathcal{H}_{1}\right) \cdot p\left(\tilde{\mathbf{y}} \mid \hat{\eta}_{\ell_{1}}, \hat{\sigma}_{\boldsymbol{\zeta}, \ell_{1}}^{2}, \mathcal{H}_{1}\right)}{p\left(\tilde{\mathbf{x}} \mid \hat{\sigma}_{\boldsymbol{\xi}, \ell_{0}}^{2}, \mathcal{H}_{0}\right) \cdot p\left(\tilde{\mathbf{y}} \mid \hat{\sigma}_{\boldsymbol{\zeta}, \ell_{0}}^{2}, \mathcal{H}_{0}\right)}
$$

Maximum likelihood estimates for the unknown variables are given by [2, Appendix 9A]:

$$
\begin{aligned}
\hat{\beta}_{\ell_{1}} & =\left(\tilde{\mathbf{s}}^{T} \tilde{\mathbf{s}}\right)^{-1} \tilde{\mathbf{s}}^{T} \tilde{\mathbf{x}} ; \hat{\eta}_{\ell_{1}}=\left(\tilde{\mathbf{d}}^{T} \tilde{\mathbf{d}}\right)^{-1} \tilde{\mathbf{d}}^{T} \tilde{\mathbf{y}} ; \\
\hat{\sigma}_{\boldsymbol{\xi}, \ell_{1}}^{2} & =\frac{1}{N M} \tilde{\mathbf{x}}^{T}\left(\mathbf{I}-\mathbf{P}_{\tilde{\mathbf{s}}}\right) \tilde{\mathbf{x}} ; \hat{\sigma}_{\boldsymbol{\xi}, \ell_{0}}^{2}=\frac{1}{N M} \tilde{\mathbf{x}}^{T} \tilde{\mathbf{x}} ; \\
\hat{\sigma}_{\boldsymbol{\zeta}, \ell_{1}}^{2} & =\frac{1}{N M} \tilde{\mathbf{y}}^{T}\left(\mathbf{I}-\mathbf{P}_{\tilde{\mathbf{d}}}\right) \tilde{\mathbf{y}} ; \hat{\sigma}_{\boldsymbol{\zeta}, \ell_{0}}^{2}=\frac{1}{N M} \tilde{\mathbf{y}}^{T} \tilde{\mathbf{y}}
\end{aligned}
$$

In the expressions above $\mathbf{P}_{\tilde{\mathbf{s}}}=\tilde{\mathbf{s}}\left(\tilde{\mathbf{s}}^{T} \tilde{\mathbf{s}}\right)^{-1} \tilde{\mathbf{s}}^{T}$ and $\mathbf{P}_{\tilde{\mathbf{d}}}=\tilde{\mathbf{d}}\left(\tilde{\mathbf{d}}^{T} \tilde{\mathbf{d}}\right)^{-1} \tilde{\mathbf{d}}^{T}$ represent projection matrices that project a vector onto the signal and noise subspaces respectively.

Making use of the MLE expressions in the ratio of Gaussian PDFs (8) results in:

$$
\begin{aligned}
L_{G}(\tilde{\mathbf{x}}, \tilde{\mathbf{y}}) & =\left(\frac{\hat{\sigma}_{\boldsymbol{\xi}, \ell_{0}}^{2}}{\hat{\sigma}_{\tilde{\xi}, \ell_{1}}^{2}}\right)^{\frac{N M}{2}} \cdot\left(\frac{\hat{\sigma}_{\tilde{\zeta}, \ell_{0}}^{2}}{\hat{\sigma}_{\tilde{\zeta}, \ell_{1}}^{2}}\right)^{\frac{N M}{2}} \\
& =\left(\frac{\tilde{\mathbf{x}}^{T} \tilde{\mathbf{x}}}{\tilde{\mathbf{x}}^{T}\left(\mathbf{I}-\mathbf{P}_{\tilde{\mathbf{s}}}\right) \tilde{\mathbf{x}}}\right)^{\frac{N M}{2}} \cdot\left(\frac{\tilde{\mathbf{y}}^{T} \tilde{\mathbf{y}}}{\tilde{\mathbf{y}}^{T}\left(\mathbf{I}-\mathbf{P}_{\tilde{\mathbf{d}}}\right) \tilde{\mathbf{y}}}\right)^{\frac{N M}{2}} \\
& =\left(\Lambda_{\tilde{x}}(\tilde{\mathbf{x}}) \cdot \Lambda_{\tilde{y}}(\tilde{\mathbf{y}})\right)^{\frac{N M}{2}}
\end{aligned}
$$

Combining this expression with (7) and noting that $(\cdot)^{\frac{N M}{2}}$ is a monotone increasing function, results in the following decision rule:

$$
\Lambda^{\prime}(\tilde{\mathbf{x}}, \tilde{\mathbf{y}})=\Lambda_{\tilde{x}}(\tilde{\mathbf{x}}) \cdot \Lambda_{\tilde{y}}(\tilde{\mathbf{y}}) \underset{\mathcal{H}_{\ell}^{(0)}}{\stackrel{\mathcal{H}_{\ell}^{(1)}}{\gtrless}} \gamma^{\prime},
$$

For a fixed probability of false alarm, the threshold $\gamma^{\prime}$ can be determined from the inverse cumulative distribution function (CDF) of the test statistic $\Lambda^{\prime}(\tilde{\mathbf{x}}, \tilde{\mathbf{y}})$, which can be acquired by means of numerical simulations or empirically estimated from training data obtained from simulations with healthy cases (distribution only under $\mathcal{H}_{\ell}^{(0)}$ is required to perform the test).

In our work we also consider an empirical test statistic which is the product of two alternative, zero shifted, test statistics:

$$
T(\tilde{\mathbf{x}}, \tilde{\mathbf{y}})=T_{\tilde{x}}(\tilde{\mathbf{x}}) \cdot T_{\tilde{y}}(\tilde{\mathbf{y}})=\frac{\tilde{\mathbf{x}}^{T} \mathbf{P}_{\tilde{\mathbf{s}}} \tilde{\mathbf{x}}}{\tilde{\mathbf{x}}^{T} \mathbf{P}_{\tilde{\mathbf{s}}}^{\perp} \tilde{\mathbf{x}}} \cdot \frac{\tilde{\mathbf{y}}^{T} \mathbf{P}_{\tilde{\mathbf{d}}} \tilde{\mathbf{y}}}{\tilde{\mathbf{y}}^{T} \mathbf{P}_{\tilde{\mathbf{d}}}^{\perp} \tilde{\mathbf{y}}} .
$$


where $\mathbf{P}^{\perp}=\mathbf{I}-\mathbf{P}$. The statistic $T_{\tilde{x}}(\tilde{\mathbf{x}})$ is derived in [5] for the microwave radar modality. We are motivated to explore the performance of the statistic $T(\tilde{\mathbf{x}}, \tilde{\mathbf{y}})$ because in our experiments the $\Lambda^{\prime}(\tilde{\mathbf{x}}, \tilde{\mathbf{y}})$ statistic is completely dominated by the $T_{\tilde{x}}(\tilde{\mathbf{x}})$ term. This occurs because our signal model $\mathbf{s}_{i}$ is much more accurate than $\mathbf{d}_{j}$.

It can be shown that $\Lambda^{\prime}(\tilde{\mathbf{x}}, \tilde{\mathbf{y}})$ can be represented in terms of $T(\tilde{\mathbf{x}}, \tilde{\mathbf{y}}), T_{\tilde{x}}(\tilde{\mathbf{x}})$ and $T_{\tilde{y}}(\tilde{\mathbf{y}})$ as:

$$
\Lambda^{\prime}(\tilde{\mathbf{x}}, \tilde{\mathbf{y}})=1+T(\tilde{\mathbf{x}}, \tilde{\mathbf{y}})+T_{\tilde{x}}(\tilde{\mathbf{x}})+T_{\tilde{y}}(\tilde{\mathbf{y}}) .
$$

This expression provides better form of comparison $\Lambda^{\prime}(\tilde{\mathbf{x}}, \tilde{\mathbf{y}})$ with $T(\tilde{\mathbf{x}}, \tilde{\mathbf{y}})$. After incorporating the constant " 1 " into the threshold, it can be seen that $\Lambda^{\prime}(\tilde{\mathbf{x}}, \tilde{\mathbf{y}})$ consists of the sum of the two separate test statistics for MR and MIT and their cross-product term. If $T_{\tilde{x}}(\tilde{\mathbf{x}})$ and $T_{\tilde{y}}(\tilde{\mathbf{y}})$ are of approximately equal power, such a combination should lead to better performance, in comparison to $T(\tilde{\mathbf{x}}, \tilde{\mathbf{y}})$, when one of the two methods fails to detect the tumour or gives false positives in substantially wrong locations.

\section{NUMERICAL SIMULATIONS}

The electromagnetic and acoustic signals were simulated using the finite-difference time-domain (FDTD) method and two-dimensional numerical breast phantoms derived from MRI images. The FDTD grid $(650$ X 650 cells) resolves the geometry depicted in Figure 1 to $0.4 \mathrm{~mm}$. For the MR technique, we use a pulsed excitation. For the MIT simulation, we use a 3-GHz continuous waveform source. The specific absorption rate is computed and used in the acoustic signal simulation [6].

Nine series of breast tissue models are considered, whose Debye properties are represented in Table 1. $\Delta_{\epsilon}$, and $\tau$ have been fixed to constant values (see the Table) while $\epsilon_{\infty}$, and $\sigma_{s}$ have been assigned with values by linearly mapping the variation of the MRI pixel intensities into the variation of $\epsilon_{\infty}$ in the range of $\epsilon_{\infty, b}(1 \pm 0.01 \mathrm{Var} / 2)$ and of $\sigma_{s}$ in the range of $\sigma_{s, b}(1 \pm 0.01 \mathrm{Var} / 2)$ with the parameters $\epsilon_{\infty, b}, \sigma_{s, b}$ taken from Table 1. Columns $\min \left(\epsilon_{r, m} / \epsilon_{r, b}\right)$, $\max \left(\epsilon_{r, m} / \epsilon_{r, b}\right), \min \left(\sigma_{m} / \sigma_{b}\right), \max \left(\sigma_{m} / \sigma_{b}\right)$ represent the minimum and maximum ratios of the resulting dielectric properties of malignant to benign tissues after mapping. The dielectric properties are evaluated using the Debye model at central frequency $6.85 \mathrm{GHz}$.

For each series, $N_{r}=10$ different realizations of tissue structure are generated, nine of which are used as the training set for the whitening procedure. On the basis of the tenth one we build two tumorous breast phantoms by placing a circular tumour $(R=3 \mathrm{~mm})$ into two positions relative to the centre of the breast: "pos.1" at [-20mm;8mm]; "pos.2" at [15mm;-15mm]. Location "pos.2" has higher density of tissue, which decreases the dielectric contrast and makes the detection task more complicated.

In order to model measurement noise, white Gaussian noise is added to the signal. Prior to the whitening procedure, the signals are pre-processed. A simple calibration procedure is used to remove the incident pulses from the MR mode signals. Artifacts in the signals due to reflections at the skin-breast boundary are removed using the minimum mean-squared-error algorithm described in [7].

\section{RESULTS AND DISCUSSION}

For each series $1, \ldots, 9$ three cases are considered (two different tumour locations and the healthy scenario). For each of these cases images of four test statistics are computed: two ('MR' and 'MIT') corresponding to the individual MR and the MIT decision rules, and two representing the outputs from the data fusion rules (the derived

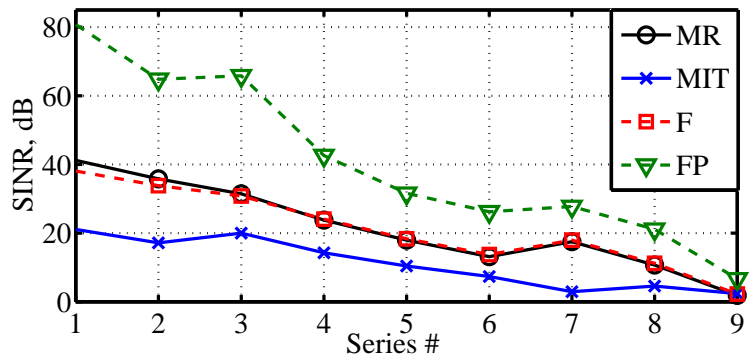

Fig. 2: SINR for "pos.1" tests. As the dielectric contrast between malignant and benign tissues drops (see Table 1), the detection performance (SINR) decreases.

test statistic in (13) denoted by "F" and the product-rule (14) denoted by "FP"). Each pixel of the images represents the value of a test statistic at locations $\left\{r_{\ell}\right\}_{\ell=1}^{L}$ on the the grid with spatial resolution of $1 \mathrm{~mm}$.

For each image we compute the signal to interference-plusnoise ratio (SINR) as the log-ratio between the maximums of the tumorous image and the corresponding healthy one: SINR = $10 \log _{10} \max \left(T_{\mathcal{H}_{1}}\right) / \max \left(T_{\mathcal{H}_{0}}\right)$.

Fig. 2 shows the SINR values for the "pos.1" tests. The SINR level drops as the contrast in dielectric properties decreases and the heterogeneity level goes up (see Table 1). The FP test statistic provides a significant improvement in the SINR along the whole range of contrast levels while the performance of $\mathrm{F}$ is biased to the MR.

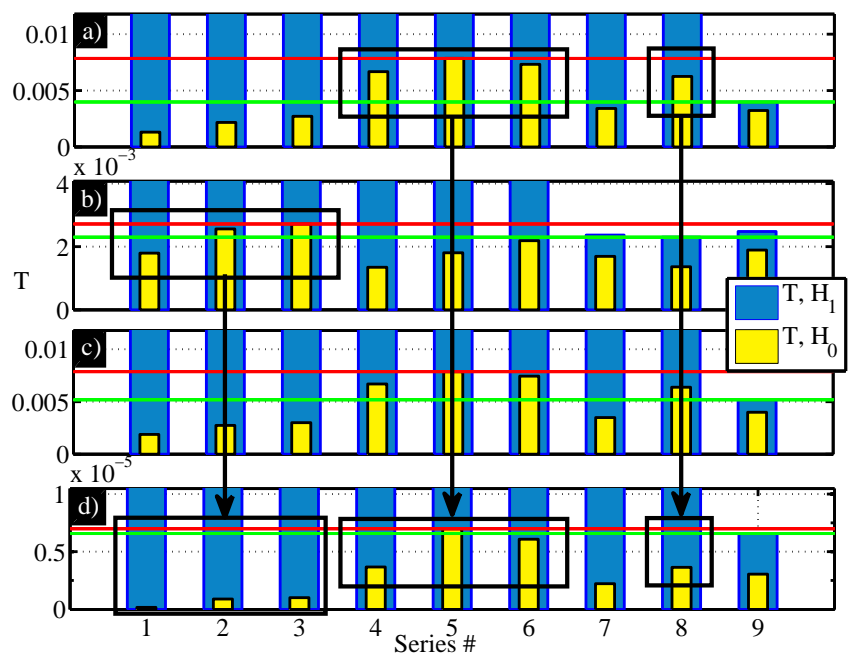

Fig. 3: Test statistic maximum values ("pos.1" case) under $\mathcal{H}_{1}$ and $\mathcal{H}_{0}$ hypotheses for each series: a) MR; b) MIT; c) F; and d) FP. Thresholds $\gamma_{\min }$ and $\gamma_{\max }$ are shown as the red and green lines. The image shows improvement in performance for FP test statistic (d) versus MR (a) and MIT(b). The cases where improvement is achieved are surrounded by black boxes.

We assess the detection performance for two different thresholds: $\gamma_{\min }$, the largest threshold such that all tumours are detected; and $\gamma_{\max }$, the smallest threshold such that there are no false alarms. Figure 3 depicts the maximum values of the test statistics obtained for the "pos.1" scenario. Two cases are represented: the tumorous (i.e., under hypothesis $\left.\mathcal{H}_{1}\right)$ and the healthy $\left(\mathcal{H}_{0}\right)$. An improved de- 
Table 1: Tissue properties for data series. Tumour is characterized by: $\epsilon_{\infty}=6.75, \sigma_{e}=0.79 \mathrm{~S} / \mathrm{m}, \Delta \epsilon=48.35, \tau=10.47 \mathrm{ps}$.

\begin{tabular}{|c|c|c|c|c|c|c|c|c|c|}
\hline \multirow{2}{*}{ Series \# } & \multicolumn{4}{|c|}{ Debye model parameters } & \multirow{2}{*}{ Var, \% } & \multirow{2}{*}{$\min \left(\epsilon_{r, m} / \epsilon_{r, b}\right)$} & \multirow{2}{*}{$\max \left(\epsilon_{r, m} / \epsilon_{r, b}\right)$} & \multirow{2}{*}{$\min \left(\sigma_{m} / \sigma_{b}\right)$} & \multirow{2}{*}{$\max \left(\sigma_{m} / \sigma_{b}\right)$} \\
\hline & $\epsilon_{\infty, b}$ & $\sigma_{s, b}$ & $\Delta_{\epsilon}$ & $\tau, \mathrm{ps}$ & & & & & \\
\hline 1 & 3.1 & 0.05 & 1.6 & 13 & 7 & 10.6 & 11.1 & 24.8 & 25.1 \\
\hline 2 & 4.0 & 0.08 & 3.5 & 13 & 30 & 6.4 & 7.7 & 11.5 & 11.9 \\
\hline 4 & 13.0 & 0.4 & 24.4 & 13 & 30 & 1.4 & 1.6 & 1.7 & 1.8 \\
\hline 5 & 13.0 & 0.4 & 24.4 & 13 & 50 & 1.3 & 1.7 & 1.7 & 1.8 \\
\hline 6 & 13.0 & 0.4 & 24.4 & 13 & 70 & 1.3 & 1.7 & 1.7 & 1.8 \\
\hline 9 & 14.2 & 0.8 & 40.5 & 13 & 10 & 1.0 & 1.1 & 1.0 & 1.0 \\
\hline
\end{tabular}

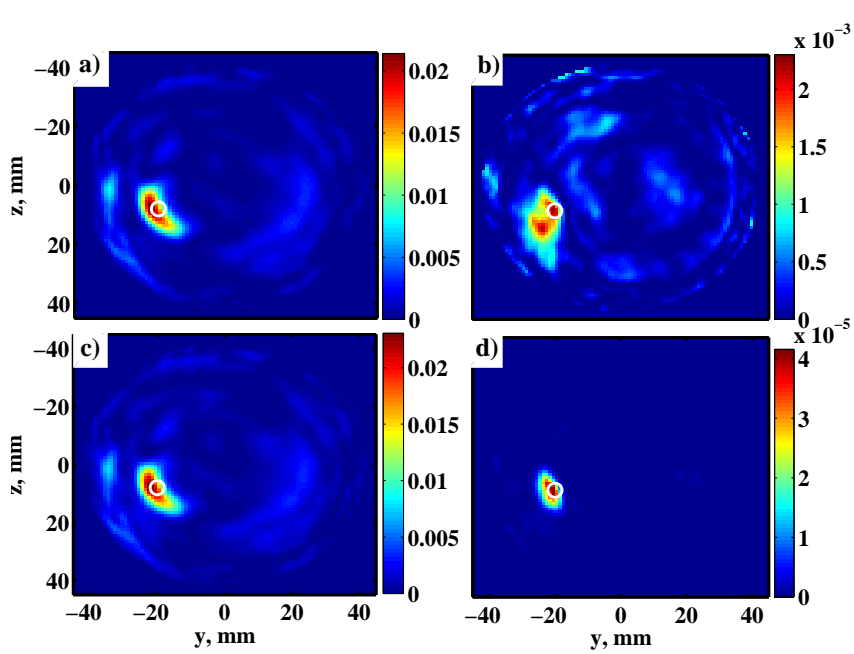

Fig. 4: Images of the test statistics for a breast model slice: a) MR; b) MIT; c) F; d) FP. True tumour location is shown by circle.

tection performance can be observed for FP with respect to MR (the $\mathcal{H}_{0}$ statistics for series 4,6 and 8 are shifted below the $\gamma_{\text {min }}$ threshold) and MIT (the $\mathcal{H}_{0}$ statistics for series 2 and 3 go below $\gamma_{\min }$ and the $\mathcal{H}_{1}$ statistics for series 7 and 8 increase dramatically). The detection capability for other series is preserved. The $\gamma_{\text {min }}$ thresholding generates four false-alarms for the MR case and two false-alarms for the MIT case, but only one false-alarm for the FP rule.

Table 2 summarizes the detection performance for both "pos.1" and "pos.2" tests. The product rule FP provides the best performance. Fig. 4 illustrates example images for the case where the FP test statistic leads to significant improvement (series \#8, "pos.1"). One may notice the difference in the spatial distribution of clutter between the MR and the MIT test statistics, which is the principle property that the fusion benefits from. Considerable suppression of the clutter may be observed for the FP test statistic. The F test statistic is dominated by the MR signal, i.e. the $T_{\tilde{x}}$ term in (15). We conjecture that this is because the MR signal template is a much better match to the simulated signal than the acoustic MIT signal template.

\section{CONCLUSIONS}

The conducted study shows that the fusion of data from microwave radar and microwave thermoacoustic measurements has the potential to lead to improved breast cancer detection performance. In the presented simulations, the performance of the derived GLRT test statis-
Table 2: Detection performance. False alarms are reported for the case when threshold $\gamma_{\min }$ is used. Misses are reported for the case when $\gamma_{\max }$ is used. Note that MR, F and FP can all achieve 1 miss and 0 false-alarms when $\gamma_{\max }$ is used.

\begin{tabular}{||l|c|c|c|c||}
\hline \hline \multirow{2}{*}{} & \multicolumn{2}{|c|}{ pos.1 } & \multicolumn{2}{c||}{ pos.2 } \\
\cline { 2 - 5 } & False alarms & Misses & False alarms & Misses \\
\hline MR & 4 & 1 & 7 & 1 \\
\hline MIT & 2 & 3 & 9 & 6 \\
\hline F & 4 & 1 & 7 & 1 \\
\hline FP & 1 & 1 & 6 & 1 \\
\hline \hline
\end{tabular}

tic is dominated by the MR signal and the heuristic test statistic performs better. We believe this can be addressed by employing a more accurate MIT signal template. Although the theoretically derived test statistic does not show significant improvement in performance over the separate MR and MIT methods, our study demonstrates that the data fusion using simple empirically derived product rule can significantly improve the overall detection performance.

\section{REFERENCES}

[1] E.C. Fear, P.M. Meaney, and M.A. Stuchly, "Microwaves for breast cancer detection?," IEEE potentials, vol. 22, no. 1, pp. 12-18, 2003.

[2] S.M. Kay, Fundamentals of Statistical Signal Processing, Volume 2: Detection Theory, Prentice Hall PTR, 1998.

[3] Ari Sihvola, Electromagnetic Mixing Formulas and Applications, Institution of Electrical Engineers, 1999.

[4] Yao Xie, Bin Guo, Jian Li, Geng Ku, and Lihong V. Wang, "Adaptive and robust methods of reconstruction for thermoacoustic tomography," IEEE Trans. Biomed. Eng., vol. 55, pp. 2741-2842, 2008.

[5] S. K. Davis, H. Tandradinata, S. C. Hagness, and B. D. van Veen, "Ultrawideband microwave breast cancer detection: a detectiontheoretic approach using the generalized likelihood ratio test," IEEE Trans. Biomed. Eng., vol. 52, no. 7, pp. 1237-1250, 2005.

[6] Guangran Zhu, Milica Popović, and Qianqian Fang, "Microwave-induced thermoacoustics: Assisting microwave tomography," IEEE Trans. Magn., vol. 45, no. 3, pp. 1654 1657, 2009.

[7] E. J. Bond, Xu Li, S. C. Hagness, and B. D. van Veen, "Microwave imaging via space-time beamforming for early detection of breast cancer," IEEE Trans. Antennas Propag., vol. 51, pp. 1690-1705, 2003. 